\title{
CONVERGENCE OF MODIFIED MULTI-STEP ITERATIVE FOR A FINITE FAMILY OF ASYMPTOTICALLY QUASI-NONEXPANSIVE MAPPINGS
}

\author{
Juan Xiao, Lei Deng, and Ming-Ge Yang
}

\begin{abstract}
In a uniformly convex Banach space, we introduce a iterative scheme for a finite family of asymptotically quasi-nonexpansive mappings and utilize a new inequality to prove several convergence results for the iterative sequence. The results generalize and unify many important known results of relevant scholars.
\end{abstract}

\section{Introduction and preliminaries}

Let $E$ be a Banach space, $K$ be a nonempty closed convex subset of $E, T$ be a self-mapping of $K$ and $F(T)$ denotes the set of fixed points of $T$.

Definition $1.1([8]) . T$ is called asymptotically nonexpansive mapping if there exists a sequence $u_{n} \in[0, \infty), \lim _{n \rightarrow \infty} u_{n}=0$, such that $\left\|T^{n} x-T^{n} y\right\| \leq$ $\left(1+u_{n}\right)\|x-y\|$ for all $n \in \mathbb{N}$ and $x, y \in K$.

Definition $1.2([16]) . T$ is called asymptotically quasi-nonexpansive mapping if there exists a sequence $u_{n} \in[0, \infty), \lim _{n \rightarrow \infty} u_{n}=0$, such that $\left\|T^{n} x-p\right\| \leq$ $\left(1+u_{n}\right)\|x-p\|$ for all $n \in \mathbb{N}$, and $x \in K, p \in F(T)$, where $F(T) \neq \varnothing$.

Definition $1.3([15]) . E$ is said to satisfy Opial's condition if for any sequence $x_{n} \in E, x_{n} \rightarrow x$ implies that $\lim \sup _{n \rightarrow \infty}\left\|x_{n}-x\right\|<\lim \sup _{n \rightarrow \infty}\left\|x_{n}-y\right\|$ for all $y \in E$ with $y \neq x$, where $x_{n} \rightarrow x$ denotes that $\left\{x_{n}\right\}$ converges weakly to $x$.

Definition 1.4 ([12]). A mapping $T$ with domain $D(T)$ and range $R(T)$ in $E$ is said to be semi-closed at $p$ if whenever $\left\{x_{n}\right\}$ is a sequence in $D(T)$ such that $\left\{x_{n}\right\}$ converges weakly to $x^{*} \in D(T)$ and $\left\{T x_{n}\right\}$ converges strongly to $p$, then $T x^{*}=p$.

Received June 9, 2013.

2010 Mathematics Subject Classification. 47H10, 26A18.

Key words and phrases. uniformly convex Banach space, fixed point, asymptotically nonexpansive mapping, asymptotically quasi-nonexpansive mapping.

Supported by National Natural Science Foundation of China (11226228), the Science and Technology Program Project of Henan Province (122300410256), the Natural Science Foundation of the Education Department of Henan Province (2011B110025). 
Definition $1.5([6])$. A finite family $\left\{T_{i}\right\}_{i=1}^{m}$ of self-mappings of $K$ is said to satisfy condition $(B)$ if there exists a nondecreasing function $f:[0, \infty) \rightarrow[0, \infty)$ with $f(0)=0, f(r)>0$ for all $r \in(0, \infty)$, such that $\max _{1 \leq i \leq m}\left\|x-T_{i} x\right\| \geq$ $f(d(x, F))$ for all $x \in K$ where $F=\bigcap_{i=1}^{m} F\left(T_{i}\right) \neq \emptyset$ and $d(x, F)=\inf _{x^{*} \in F} \| x-$ $x^{*} \|$.

In [1], [3] and [6], authors introduced several Modified multi-step iterations weakly and strongly converges to a common fixed point for a finite family of nonexpansive mappings or asymptotically quasi-nonexpansive mappings.

In [13], the author introduced the Modified multi-step iteration and proved that the sequence $\left\{x_{n}\right\}$ defined by (1) converges to a common fixed point of a continuous and strongly pseudocontractive operator in Banach spaces.

$$
\left\{\begin{aligned}
x_{1} & \in E, \\
x_{n+1} & =\left(1-\beta_{n}^{1}\right) x_{n}+\beta_{n}^{1} T y_{n}^{1}, \\
y_{n}^{i} & =\left(1-\beta_{n}^{i+1}\right) x_{n}+\beta_{n}^{i+1} T y_{n}^{i+1}, i=1,2, \ldots, m-2, \\
y_{n}^{m-1} & =\left(1-\beta_{n}^{m}\right) x_{n}+\beta_{n}^{m} T x_{n}, m \geq 2,
\end{aligned}\right.
$$

where the sequences $\left\{\beta_{n}^{i}\right\}_{n=1}^{\infty} \subseteq[0,1], i=1,2, \ldots, m$ satisfy certain conditions.

In this paper, we generalize the iterative processes (1) to the following iterative process:

Let $K$ be a nonempty closed convex subset of a Banach space $E$ and $T_{1}, T_{2}$, $\ldots, T_{m}: K \rightarrow K$ be a finite family of asymptotically quasi-nonexpansive mappings. Then, the iterative sequence $\left\{x_{n}\right\}$ defined by the iterative scheme:

$$
\left\{\begin{aligned}
x_{j} & \in E(j=1,2, \ldots, r), \\
x_{n+1} & =\left(1-\beta_{n}^{1}\right) x_{n-q_{1}}+\beta_{n}^{1} T_{1}^{n} y_{n}^{1} \\
y_{n}^{i} & =\left(1-\beta_{n}^{i+1}\right) x_{n-q_{i+1}}+\beta_{n}^{i+1} T_{i+1}^{n} y_{n}^{i+1}, i=1,2, \ldots, m-2, \\
y_{n}^{m-1} & =\left(1-\beta_{n}^{m}\right) x_{n-q_{m}}+\beta_{n}^{m} T_{m}^{n} x_{n}, m \geq 2, n \geq r,
\end{aligned}\right.
$$

where $\left\{\beta_{n}^{i}\right\}_{i=1}^{m} \subset[\delta, 1-\delta]$ with $\delta \in(0,1),\left\{q_{i}\right\}_{i=1}^{m}$ is a nonnegative integer sequence in $[0, r]$ and $r, m \in \mathbb{N}$ are fixed numbers.

Remark 1. In (2), taking $r=1,\left\{T_{i}^{n}\right\}_{i=1}^{m}=T$ and $\left\{q_{i}\right\}_{i=1}^{m}=0$ for all $n \geq 1$, then we get (1). So the sequence $\left\{x_{n}\right\}$ defined by (2) extend the sequence $\left\{x_{n}\right\}$ defined by (1).

Remark 2. The class of asymptotically quasi-nonexpansive mappings is a generalization of the class of nonexpansive mappings and asymptotically nonexpansive mappings.

In a uniformly convex Banach spaces, we introduce a new inequality and prove that the sequence $\left\{x_{n}\right\}$ defined by (2) weakly and strongly converges to a common fixed point for a finite family of asymptotically quasi-nonexpansive mappings, finally we obtain several corollaries. Our results generalize and unify the corresponding results of relevant scholars $[1,2,3,4,5,6,10,13,20]$. 


\section{Lemmas}

We need the following lemmas to prove our main results.

Lemma 1 ([1]). Let $\left\{a_{n}\right\}_{n=1}^{\infty}$ and $\left\{l_{n}\right\}_{n=1}^{\infty}$ be two nonnegative real sequences satisfying

$$
a_{n+1} \leq\left(1+l_{n}\right) a_{n}, \quad \forall n \in \mathbb{N} .
$$

where $\sum_{n=1}^{\infty} l_{n}<+\infty$, then $\lim _{n \rightarrow \infty} a_{n}$ exists.

Lemma 2. Let $\left\{a_{n}\right\}$ and $\left\{l_{n}^{(i)}\right\}_{i=0}^{m-1}$ be sequences of nonnegative real numbers such that

$$
a_{n+1} \leq l_{n}^{(0)} a_{n}+\sum_{i=1}^{m} l_{n}^{(i)} a_{n-q_{i}}, \quad(n \geq r)
$$

where $\left\{q_{i}\right\}_{i=1}^{m}$ is a nonnegative integer sequence in $[0, r]$ and $r, m \in \mathbb{N}$ are fixed numbers. If $\liminf \operatorname{in}_{n \rightarrow \infty} l_{n}^{(0)}>0$ and $\sum_{n=1}^{\infty} c_{n}<\infty$, where

$$
c_{n}= \begin{cases}0, & \sum_{i=0}^{m} l_{n}^{(i)} \leq 1 \\ \sum_{i=0}^{m} l_{n}^{(i)}-1, & \sum_{i=0}^{m} l_{n}^{(i)}>1\end{cases}
$$

then $\lim _{n \rightarrow \infty} a_{n}$ exists.

Proof. Let $\left\{b_{n}\right\}_{n=1}^{\infty}$ be a sequence defined by

$$
b_{n}= \begin{cases}a_{n}, & n=1,2, \ldots, r \\ \max \left\{a_{n-r}, a_{n-r+1}, \ldots, a_{n}\right\}, & n=r+1, r+2, \ldots\end{cases}
$$

Using (3), we obtain

$$
\begin{aligned}
a_{n+1} & \leq l_{n}^{(0)} a_{n}+\sum_{i=1}^{m} l_{n}^{(i)} a_{n-q_{i}} \leq l_{n}^{(0)} b_{n}+\sum_{i=1}^{m} l_{n}^{(i)} b_{n} \\
& \leq\left(1+c_{n}\right) b_{n}, n=r+1, r+2, \ldots
\end{aligned}
$$

Thus,

$$
\begin{aligned}
b_{n+1} & =\max \left\{a_{n-r+1}, a_{n-r+2}, \ldots, a_{n+1}\right\} \\
& \leq \max \left\{a_{n-r}, a_{n-r+1}, a_{n-r+2}, \ldots, a_{n}, a_{n+1}\right\} \\
& \leq \max \left\{b_{n}, a_{n+1}\right\} \\
& \leq\left(1+c_{n}\right) b_{n}, n=r+1, r+2, \ldots
\end{aligned}
$$

Since $\sum_{n=1}^{\infty} c_{n}<\infty$, by Lemma 1 , we obtain $\lim _{n \rightarrow \infty} b_{n}$ exists.

Suppose $\lim _{n \rightarrow \infty} b_{n}=a$. Next, we will prove $\left\{a_{n}\right\}_{n=1}^{\infty}$ also converges to $a$.

By the definition of $\left\{b_{n}\right\}_{n=1}^{\infty}$, we get $a_{n} \leq b_{n}$ for all $n \in \mathbb{N}$. If $a=0$, then it is easy to obtain $\left\{a_{n}\right\}$ also converges to 0 . If $a \neq 0$, suppose that $\left\{a_{n}\right\}_{n=1}^{\infty}$ does not converge to $a$. Since $\lim _{n \rightarrow \infty} b_{n}=a$, it is easy to find $\xi>0$ such that for all $j>0$, there exists $n_{j}>j$ satisfying

$$
a_{n_{j}}<a-\xi
$$


As $\liminf _{n \rightarrow \infty} l_{n}^{(0)}>0$, then there exist $\theta \in(0,1)$ and $N>0$ such that for all $n \geq N$

$$
l_{n}^{(0)} \geq \theta
$$

Let $\varepsilon=\min \left\{\frac{(1-\theta) \theta^{r} \xi}{2-\theta-\theta^{r}}, 2 a\right\}$. By $\lim _{n \rightarrow \infty} b_{n}=a$ and $\lim _{n \rightarrow \infty} c_{n}=0$, then there exists $N_{\varepsilon}>N$ such that for all $n \geq N_{\varepsilon}$

$$
a-\frac{\varepsilon}{4}<b_{n}<a+\frac{\varepsilon}{4}, \quad c_{n}<\frac{\varepsilon}{2 a} \leq 1 .
$$

By (4), there exists $n_{0} \geq N_{\varepsilon}+2 r+1\left(n_{0}-r \geq N_{\varepsilon}+r+1\right)$, such that $a_{n_{0}-r}<a-\xi$. From (3), (5) and (6), we have

$$
\begin{aligned}
a_{n_{0}-r+1} & \leq l_{n_{0}-r}^{(0)} a_{n_{0}-r}+\sum_{i=1}^{m} l_{n_{0}-r}^{(i)} a_{n_{0}-r-q_{i}} \\
& <l_{n_{0}-r}^{(0)}(a-\xi)+\sum_{i=1}^{m} l_{n_{0}-r}^{(i)}\left(a+\frac{\varepsilon}{4}\right) \\
& <\left(1+c_{n_{0}-r}\right) a-l_{n_{0}-r}^{(0)} \xi+\left(1+c_{n_{0}-r}\right) \frac{\varepsilon}{4} \\
& <a-\theta \xi+\varepsilon .
\end{aligned}
$$

Further, by (7), we obtain

$$
\begin{aligned}
a_{n_{0}-r+2} & \leq l_{n_{0}-r+1}^{(0)} a_{n_{0}-r+1}+\sum_{i=1}^{m} l_{n_{0}-r+1}^{(i)} a_{n_{0}-r+1-q_{i}} \\
& <l_{n_{0}-r+1}^{(0)}(a-\theta \xi+\varepsilon)+\sum_{i=1}^{m} l_{n_{0}-r+1}^{(i)}\left(a+\frac{\varepsilon}{4}\right) \\
& <\left(1+c_{n_{0}-r+1}\right) a-l_{n_{0}-r+1}^{(0)}(\theta \xi-\varepsilon)+\left(1+c_{n_{0}-r+1}\right) \frac{\varepsilon}{4} \\
& <a-\theta^{2} \xi+(\theta+1) \varepsilon .
\end{aligned}
$$

Continuously, we can prove that

$$
a_{n_{0}-r+i}<a-\theta^{i} \xi+\left(\frac{1-\theta^{i}}{1-\theta}\right) \varepsilon, i=0,1, \ldots, r .
$$

It follows from $(8)$ and $\varepsilon \leq \frac{(1-\theta) \theta^{r} \xi}{2-\theta-\theta^{r}}$ that

$$
\begin{aligned}
a_{n_{0}-r+i} & <a-\theta^{i} \xi+\left(\frac{1-\theta^{i}}{1-\theta}\right) \varepsilon \\
& \leq a-\theta^{i} \frac{2-\theta-\theta^{r}}{(1-\theta) \theta^{r}} \varepsilon+\left(\frac{1-\theta^{i}}{1-\theta}\right) \varepsilon \\
& =a-\frac{2-\theta-\theta^{r-i}}{(1-\theta) \theta^{r-i}} \varepsilon
\end{aligned}
$$




$$
\begin{aligned}
& =a-\frac{(1-\theta) \theta^{r-i}+(2-\theta)\left(1-\theta^{r-i}\right)}{(1-\theta) \theta^{r-i}} \varepsilon \\
& \leq a-\varepsilon, i=0,1, \ldots, r .
\end{aligned}
$$

By the definition of $\left\{b_{n}\right\}$, we have $b_{n_{0}}<a-\varepsilon$, which contradicts $b_{n_{0}}>a-\frac{\varepsilon}{4}$. Thus $\left\{a_{n}\right\}_{n=1}^{\infty}$ converges to $a$.

Lemma 3. Let $E$ be a Banach space, $K$ be a nonempty closed convex subset of $E,\left\{T_{i}\right\}_{i=1}^{m}$ be a finite family of asymptotically quasi-nonexpansive selfmappings of $K$ with sequences $\left\{u_{n}^{i}\right\}_{i=1}^{m} \subset[0, \infty)$ such that $\sum_{n=1}^{\infty} u_{n}^{i}<+\infty$. Let $\left\{x_{n}\right\}$ be the sequence as defined by $(2)$ satisfying $\left\{\beta_{n}^{i}\right\}_{i=1}^{m} \subset[\delta, 1-\delta]$ with $\delta \in(0,1)$. Then $\lim _{n \rightarrow \infty}\left\|x_{n}-x^{*}\right\|$ and $\lim _{n \rightarrow \infty}\left\|y_{n}^{i}-x^{*}\right\|$ are existent and equal for all $x^{*} \in F$ and $i=1,2, \ldots, m-1$.

Proof. Let $x^{*} \in F=\bigcap_{i=1}^{m} F\left(T_{i}\right) \neq \emptyset, v_{n}=\max _{1 \leq i \leq m} u_{n}^{i}$ for each $n$. Since $\sum_{n=1}^{\infty} u_{n}^{i}<+\infty$ for each $i$, therefore $\sum_{n=1}^{\infty} v_{n}<+\infty$. Put

$$
\beta_{n}^{(i)}= \begin{cases}\beta_{n}^{1} \beta_{n}^{2} \cdots \beta_{n}^{m}, & i=0 ; \\ \beta_{n}^{1} \beta_{n}^{2} \cdots\left(1-\beta_{n}^{i}\right), & i=1,2, \ldots, m .\end{cases}
$$

It follows from (2), we obtain that for any $i=1,2, \ldots, m-2$

$$
\begin{aligned}
\left\|y_{n}^{i}-x^{*}\right\| & =\left\|\left(1-\beta_{n}^{i+1}\right) x_{n-q_{i+1}}+\beta_{n}^{i+1} T_{i+1}^{n} y_{n}^{i+1}-x^{*}\right\| \\
& \leq\left(1-\beta_{n}^{i+1}\right)\left\|x_{n-q_{i+1}}-x^{*}\right\|+\beta_{n}^{i+1}\left(1+u_{n}^{i+1}\right)\left\|y_{n}^{i+1}-x^{*}\right\| \\
& \leq\left(1+v_{n}\right)\left(\left(1-\beta_{n}^{i+1}\right)\left\|x_{n-q_{i+1}}-x^{*}\right\|+\beta_{n}^{i+1}\left\|y_{n}^{i+1}-x^{*}\right\|\right),
\end{aligned}
$$

and for $i=m-1$, we have

$$
\begin{aligned}
\left\|y_{n}^{m-1}-x^{*}\right\| & =\left\|\left(1-\beta_{n}^{m}\right) x_{n-q_{m}}+\beta_{n}^{m} T_{m}^{n} x_{n}-x^{*}\right\| \\
& \leq\left(1-\beta_{n}^{m}\right)\left\|x_{n-q_{m}}-x^{*}\right\|+\beta_{n}^{m}\left(1+u_{n}^{m}\right)\left\|x_{n}-x^{*}\right\| \\
& \leq\left(1+v_{n}\right)\left(\left(1-\beta_{n}^{m}\right)\left\|x_{n-q_{m}}-x^{*}\right\|+\beta_{n}^{m}\left\|x_{n}-x^{*}\right\|\right) .
\end{aligned}
$$

Then, from (2), (9) and (10), we get

$$
\begin{aligned}
\left\|x_{n+1}-x^{*}\right\| \leq & \left(1-\beta_{n}^{1}\right)\left\|x_{n-q_{1}}-x^{*}\right\|+\beta_{n}^{1}\left\|T_{1}^{n} y_{n}^{1}-x^{*}\right\| \\
\leq & \left(1+v_{n}\right)\left(\left(1-\beta_{n}^{1}\right)\left\|x_{n-q_{1}}-x^{*}\right\|+\beta_{n}^{1}\left\|y_{n}^{1}-x^{*}\right\|\right) \\
\leq & \left(1+v_{n}\right)^{2}\left(\left(1-\beta_{n}^{1}\right)\left\|x_{n-q_{1}}-x^{*}\right\|+\beta_{n}^{1}\left(1-\beta_{n}^{2}\right)\left\|x_{n-q_{2}}-x^{*}\right\|\right. \\
& \left.+\beta_{n}^{1} \beta_{n}^{2}\left\|y_{n}^{2}-x^{*}\right\|\right) \\
& \vdots \\
\leq & \left(1+v_{n}\right)^{m-1}\left(\sum_{i=1}^{m-1} \beta_{n}^{(i)}\left\|x_{n-q_{i}}-x^{*}\right\|\right. \\
& \left.+\beta_{n}^{1} \beta_{n}^{2} \cdots \beta_{n}^{m-1}\left\|y_{n}^{m-1}-x^{*}\right\|\right)
\end{aligned}
$$




$$
\begin{aligned}
& \leq\left(1+v_{n}\right)^{m}\left(\beta_{n}^{(0)}\left\|x_{n}-x^{*}\right\|+\sum_{i=1}^{m} \beta_{n}^{(i)}\left\|x_{n-q_{i}}-x^{*}\right\|\right) \\
& =l_{n}^{(0)}\left\|x_{n}-x^{*}\right\|+\sum_{i=1}^{m} l_{n}^{(i)}\left\|x_{n-q_{i}}-x^{*}\right\|,
\end{aligned}
$$

where $l_{n}^{(i)}=\left(1+v_{n}\right)^{m} \beta_{n}^{(i)}$. By the definition of $\left\{\beta_{n}^{(i)}\right\}$, we obtain $l_{n}^{(0)}=(1+$ $\left.v_{n}\right)^{m} \beta_{n}^{(0)} \geq \delta^{m}$ for some $\delta \in(0,1)$, then we have $\liminf _{n \rightarrow \infty} l_{n}^{(0)}>0$. Let $\left\{c_{n}\right\}_{n=1}^{\infty}$ be a sequence defined by

$$
c_{n}= \begin{cases}0, & \sum_{i=0}^{m} l_{n}^{(i)} \leq 1 \\ \sum_{i=0}^{m} l_{n}^{(i)}-1, & \sum_{i=0}^{m} l_{n}^{(i)}>1 .\end{cases}
$$

Since $\sum_{i=0}^{m} \beta_{n}^{(i)}=1$ and $\sum_{n=1}^{\infty} v_{n}<+\infty$, then

$$
\begin{aligned}
\sum_{n=1}^{\infty} c_{n} & =\sum_{n=1}^{\infty}\left(\sum_{i=0}^{m} l_{n}^{(i)}-1\right) \\
& =\sum_{n=1}^{\infty}\left(\left(1+v_{n}\right)^{m}-1\right) \\
& =\sum_{n=1}^{\infty}\left(C_{m}^{1} v_{n}+C_{m}^{2} v_{n}^{2}+\cdots+C_{m}^{m} v_{n}^{m}\right)<+\infty
\end{aligned}
$$

It follows from Lemma 2 together with (11) and (12), we obtain

$$
\lim _{n \rightarrow \infty}\left\|x_{n}-x^{*}\right\|
$$

exists for all $x^{*} \in F$. Moreover, $\lim _{n \rightarrow \infty}\left\|x_{n}-x^{*}\right\|$ exists together with (11), it is easy to see that $\lim _{n \rightarrow \infty}\left\|y_{n}^{i}-x^{*}\right\|$ also exist for all $i=1,2, \ldots, m-1$ and $\lim _{n \rightarrow \infty}\left\|x_{n}-x^{*}\right\|=\lim _{n \rightarrow \infty}\left\|y_{n}^{i}-x^{*}\right\|$.

Lemma 4 ([17]). Let $p>1, r>0$ be two fixed numbers and $E$ be a Banach space. Then $E$ is uniformly convex if and only if there exists a continuous, strictly increasing, and convex function $g:[0, \infty) \rightarrow[0, \infty)$ with $g(0)=0$ such that $\|\lambda x+(1-\lambda) y\|^{p} \leq \lambda\|x\|^{p}+(1-\lambda)\|y\|^{p}-\omega_{p}(\lambda) g(\|x-y\|)$ for all $x, y \in$ $B_{r}(0)=\{x \in E:\|x\| \leq r\}$, and $\lambda \in[0,1]$, where $\omega_{p}(\lambda)=\lambda(1-\lambda)^{p}+(1-\lambda) \lambda^{p}$.

Lemma 5 ([14]). Let $E$ be a uniformly convex Banach space, $K$ be a nonempty closed subset of $E$, and $T: K \rightarrow K$ an asymptotically nonexpansive mapping. Then $I-T$ is semi-closed at zero, i.e., for each sequence $\left\{x_{n}\right\} \subset K$, if $\left\{x_{n}\right\}$ converges weakly to $p \in K$ and $\left\{(I-T) x_{n}\right\}$ converges strongly to 0 , then $p \in F(T)$.

Lemma 6 ([15]). Let $E$ be a Banach space which satisfies Opial's condition and let $\left\{x_{n}\right\}$ be a sequence in $E$. Let $u, v \in E$ be such that $\lim _{n \rightarrow \infty}\left\|x_{n}-u\right\|$ and 
$\lim _{n \rightarrow \infty}\left\|x_{n}-v\right\|$ exist. If $\left\{x_{n_{k}}\right\}$ and $\left\{x_{m_{k}}\right\}$ are subsequences of $\left\{x_{n}\right\}$ which converge weakly to $u$ and $v$, respectively, then $u=v$.

\section{Main results}

In this section, we will prove our main theorems.

Theorem 1. Let $E$ be a Banach space, $K$ be a nonempty closed convex subset of $E,\left\{T_{i}\right\}_{i=1}^{m}$ and $\left\{x_{n}\right\}$ as taken in Lemma 3. Then the sequence $\left\{x_{n}\right\}_{n=1}^{\infty}$ be given by (2) converges strongly to a common fixed point of $\left\{T_{i}\right\}_{i=1}^{m}$ if and only if $\liminf \operatorname{in}_{n \rightarrow \infty} d\left(x_{n}, F\right)=0$, where $d\left(x_{n}, F\right)=\inf _{p \in F}\left\|x_{n}-p\right\|$.

Proof. Necessity is obvious. We only prove the sufficiency. Suppose

$$
\liminf _{n \rightarrow \infty} d\left(x_{n}, F\right)=0 \text {. }
$$

As proved in Lemma 3, we obtain $\lim _{n \rightarrow \infty}\left\|x_{n}-x^{*}\right\|$ exists for all $x^{*} \in F$. This further implies that $\lim _{n \rightarrow \infty} d\left(x_{n}, F\right)$ exists.

By the fact that $\liminf _{n \rightarrow \infty} d\left(x_{n}, F\right)=0$, we obtain $\lim _{n \rightarrow \infty} d\left(x_{n}, F\right)=0$, that is

$$
\lim _{n \rightarrow \infty} d\left(x_{n}, F\right)=\lim _{n \rightarrow \infty} \inf _{x^{*} \in F}\left\|x_{n}-x^{*}\right\|=0
$$

It implies that

$$
\inf _{x^{*} \in F} \lim _{n \rightarrow \infty}\left\|x_{n}-x^{*}\right\|=0 .
$$

So for any given $\varepsilon>0$, there exist $p \in F$ and $N>0$ such that

$$
\left\|x_{n}-p\right\|<\frac{\varepsilon}{2}
$$

for all $n>N$. This shows that

$$
\left\|x_{n+m}-x_{n}\right\| \leq\left\|x_{n+m}-p\right\|+\left\|x_{n}-p\right\|<\frac{\varepsilon}{2}+\frac{\varepsilon}{2}=\varepsilon
$$

for all $n>N$ and $m \geq 0$. Hence $\left\{x_{n}\right\}$ is a Cauchy sequence. Since $E$ is a complete, we can obtain $\left\{x_{n}\right\}$ is convergent. Let $\lim _{n \rightarrow \infty} x_{n}=x^{\prime}$. There exists $N^{\prime}>0$ such that

$$
\left\|x_{n}-x^{\prime}\right\|<\frac{\varepsilon}{2}
$$

for all $n>N^{\prime}$. Let $N^{*}=\max \left\{N, N^{\prime}\right\}$. For all $n \geq N^{*}$, we have

$$
\begin{aligned}
\left\|T_{i} x^{\prime}-x^{\prime}\right\| & \leq\left\|T_{i} x^{\prime}-T_{i} p\right\|+\left\|p-x^{\prime}\right\| \\
& \leq\left(1+v_{1}\right)\left\|p-x^{\prime}\right\|+\left\|p-x^{\prime}\right\| \\
& \leq\left(2+v_{1}\right)\left(\left\|x_{n}-p\right\|+\left\|x^{\prime}-x_{n}\right\|\right) \\
& \leq\left(2+v_{1}\right) \varepsilon
\end{aligned}
$$

for any $i=1,2, \ldots, m$. By the arbitrariness of $\varepsilon$, it gets $\left\|T_{i} x^{\prime}-x^{\prime}\right\|=0$ for any $i=1,2, \ldots, m$. So the sequence $\left\{a_{n}\right\}_{n=1}^{\infty}$ converges strongly to $x^{\prime} \in F$. 
Theorem 2. Let $E$ be a uniformly convex Banach space, $K$ be a nonempty closed convex subset of $E,\left\{T_{i}\right\}_{i=1}^{m}$ be a family of asymptotically quasi-nonexpansive self-mappings of $K$ with sequences $\left\{u_{n}^{i}\right\}_{i=1}^{m} \subset[0, \infty)$ such that $\sum_{n=1}^{\infty} u_{n}^{i}<$ $+\infty$. If the iteration sequence $\left\{x_{n}\right\}$ is defined by (2) satisfying $\left\{\beta_{n}^{i}\right\}_{i=1}^{m} \subset$ $[\delta, 1-\delta]$ with $\delta \in(0,1)$, then $\lim _{n \rightarrow \infty}\left\|x_{n}-T_{i} x_{n}\right\|=0$.

Proof. Let $x^{*}$ be a common fixed point of $\left\{T_{i}\right\}_{i=1}^{m}, v_{n}=\max _{1 \leq i \leq m} u_{n}^{i}$ for each $n$. Since $\sum_{n=1}^{\infty} u_{n}^{i}<+\infty$ for each $i$, therefore $\sum_{n=1}^{\infty} v_{n}<+\infty$. Since $E$ is uniformly convex Banach space, from Lemma 4 , let $p=2$, we get

$$
\begin{aligned}
\left\|x_{n+1}-x^{*}\right\|^{2}= & \left\|\left(1-\beta_{n}^{1}\right) x_{n-q_{1}}+\beta_{n}^{1} T_{1}^{n} y_{n}^{1}-x^{*}\right\|^{2} \\
= & \left\|\left(1-\beta_{n}^{1}\right)\left(x_{n-q_{1}}-x^{*}\right)+\beta_{n}^{1}\left(T_{1}^{n} y_{n}^{1}-x^{*}\right)\right\|^{2} \\
\leq & \left(1-\beta_{n}^{1}\right)\left\|x_{n-q_{1}}-x^{*}\right\|^{2}+\beta_{n}^{1}\left\|T_{1}^{n} y_{n}^{1}-x^{*}\right\|^{2} \\
& -\left(\beta_{n}^{1}\left(1-\beta_{n}^{1}\right)^{2}+\left(1-\beta_{n}^{1}\right) \beta_{n}^{12}\right) g\left(\left\|T_{1}^{n} y_{n}^{1}-x_{n-q_{1}}\right\|\right) \\
\leq & \left(1-\beta_{n}^{1}\right)\left\|x_{n-q_{1}}-x^{*}\right\|^{2}+\beta_{n}^{1}\left(1+v_{n}\right)\left\|y_{n}^{1}-x^{*}\right\|^{2} \\
& -2 \delta^{3} g\left(\left\|T_{1}^{n} y_{n}^{1}-x_{n-q_{1}}\right\|\right)
\end{aligned}
$$

and

$$
\begin{aligned}
\left\|y_{n}^{i}-x^{*}\right\|^{2}= & \left\|\left(1-\beta_{n}^{i+1}\right) x_{n-q_{i+1}}+\beta_{n}^{i+1} T_{i+1}^{n} y_{n}^{i+1}-x^{*}\right\|^{2} \\
\leq & \left(1-\beta_{n}^{i+1}\right)\left\|x_{n-q_{i+1}}-x^{*}\right\|^{2}+\beta_{n}^{i+1}\left\|T_{i+1}^{n} y_{n}^{i+1}-x^{*}\right\|^{2} \\
& -\omega_{2}\left(\beta_{n}^{i+1}\right) g\left(\left\|T_{i+1}^{n} y_{n}^{i+1}-x_{n-q_{i+1}}\right\|\right) \\
\leq & \left(1-\beta_{n}^{i+1}\right)\left\|x_{n-q_{i+1}}-x^{*}\right\|^{2}+\beta_{n}^{i+1}\left(1+v_{n}\right)\left\|y_{n}^{i+1}-x^{*}\right\|^{2} \\
& -\omega_{2}\left(\beta_{n}^{i+1}\right) g\left(\left\|T_{i+1}^{n} y_{n}^{i+1}-x_{n-q_{i+1}}\right\|\right) \\
\leq & \left(1-\beta_{n}^{i+1}\right)\left\|x_{n-q_{i+1}}-x^{*}\right\|^{2}+\beta_{n}^{i+1}\left(1+v_{n}\right)\left\|y_{n}^{i+1}-x^{*}\right\|^{2} \\
& -2 \delta^{3} g\left(\left\|T_{i+1}^{n} y_{n}^{i+1}-x_{n-q_{i+1}}\right\|\right)
\end{aligned}
$$

for any $i=1,2, \ldots, m-2$ and

$$
\begin{aligned}
\left\|y_{n}^{m-1}-x^{*}\right\|^{2}= & \left\|\left(1-\beta_{n}^{m}\right) x_{n-q_{m}}+\beta_{n}^{m} T_{m}^{n} x_{n}-x^{*}\right\|^{2} \\
\leq & \left(1-\beta_{n}^{m}\right)\left\|x_{n-q_{m}}-x^{*}\right\|^{2}+\beta_{n}^{m}\left(1+v_{n}\right)\left\|x_{n}-x^{*}\right\|^{2} \\
& -\omega_{2}\left(\beta_{n}^{m}\right) g\left(\left\|T_{m}^{n} x_{n}-x_{n-q_{m}}\right\|\right) \\
\leq & \left(1-\beta_{n}^{m}\right)\left\|x_{n-q_{m}}-x^{*}\right\|^{2}+\beta_{n}^{m}\left(1+v_{n}\right)\left\|x_{n}-x^{*}\right\|^{2} \\
& -2 \delta^{3} g\left(\left\|T_{m}^{n} x_{n}-x_{n-q_{m}}\right\|\right) .
\end{aligned}
$$

By (13), we have

$$
\begin{aligned}
2 \delta^{3} g\left(\left\|T_{1}^{n} y_{n}^{1}-x_{n-q_{1}}\right\|\right) \leq & \left(1-\beta_{n}^{1}\right)\left(\left\|x_{n-q_{1}}-x^{*}\right\|^{2}-\left\|x_{n+1}-x^{*}\right\|^{2}\right) \\
& +\beta_{n}^{1}\left(\left(1+v_{n}\right)\left\|y_{n}^{1}-x^{*}\right\|^{2}-\left\|x_{n+1}-x^{*}\right\|^{2}\right) .
\end{aligned}
$$


Using Lemma 3, we have $\lim _{n \rightarrow \infty}\left\|x_{n}-x^{*}\right\|$ and $\lim _{n \rightarrow \infty}\left\|y_{n}^{i}-x^{*}\right\|$ are existent and equal. Hence, by (16) and $\lim _{n \rightarrow \infty} v_{n}=0$, we obtain

$$
g\left(\left\|T_{1}^{n} y_{n}^{1}-x_{n-q_{1}}\right\|\right) \rightarrow 0 \quad(\text { as } n \rightarrow \infty) .
$$

But $g$ is strictly increasing, continuous and $g(0)=0$. Therefore

$$
\left\|T_{1}^{n} y_{n}^{1}-x_{n-q_{1}}\right\| \rightarrow 0 \quad(\text { as } n \rightarrow \infty) .
$$

Further, similar to the computations above, using (14) and (15), we also can get for any $i=1,2, \ldots, m-2$

$$
\left\|T_{i+1}^{n} y_{n}^{i+1}-x_{n-q_{i+1}}\right\| \rightarrow 0 \quad(\text { as } n \rightarrow \infty),
$$

and

$$
\left\|T_{m}^{n} x_{n}-x_{n-q_{m}}\right\| \rightarrow 0 \quad(\text { as } n \rightarrow \infty) .
$$

It follows from (2) and (17) that

$$
\left\|x_{n+1}-x_{n-q_{1}}\right\|=\beta_{n}^{1}\left\|T_{1}^{n} y_{n}^{1}-x_{n-q_{1}}\right\| \rightarrow 0 \quad(\text { as } n \rightarrow \infty) .
$$

This implies that

(21) $\quad\left\|x_{n+1}-x_{n}\right\|=0,\left\|x_{n}-x_{n-q_{i}}\right\| \rightarrow 0 \quad($ as $n \rightarrow \infty), i=1,2, \ldots, m$.

Notice that from (2), (17), (18) and (21), for any $i=1,2, \ldots, m-2$, we have

$$
\left\|x_{n}-y_{n}^{i}\right\|=\left\|x_{n}-\left(\left(1-\beta_{n}^{i+1}\right) x_{n-q_{i+1}}+\beta_{n}^{i+1} T_{i+1}^{n} y_{n}^{i+1}\right)\right\|
$$

$$
\leq\left\|x_{n}-x_{n-q_{i+1}}\right\|+\beta_{n}^{i+1}\left\|x_{n-q_{i+1}}-T_{i+1}^{n} y_{n}^{i+1}\right\| \rightarrow 0(\text { as } n \rightarrow \infty),
$$

and it follows from (2), (19) and (21) that for $i=m-1$

$$
\left\|x_{n}-y_{n}^{m-1}\right\|=\left\|x_{n}-\left(\left(1-\beta_{n}^{m}\right) x_{n-q_{m}}+\beta_{n}^{m} T_{m}^{n} x_{n}\right)\right\|
$$

$$
\leq\left\|x_{n}-x_{n-q_{m}}\right\|+\beta_{n}^{m}\left\|x_{n-q_{m}}-T_{m}^{n} x_{n}\right\| \rightarrow 0 \quad(\text { as } n \rightarrow \infty) .
$$

It follows from (17), (18), (21) and (22), for $i=1,2, \ldots, m-1$

$$
\begin{aligned}
\left\|T_{i}^{n} x_{n}-x_{n}\right\| & \leq\left\|T_{i}^{n} x_{n}-T_{i}^{n} y_{n}^{i}\right\|+\left\|T_{i}^{n} y_{n}^{i}-x_{n-q_{i}}\right\|+\left\|x_{n-q_{i}}-x_{n}\right\| \\
& \leq\left(1+v_{n}\right)\left\|x_{n}-y_{n}^{i}\right\|+\left\|T_{i}^{n} y_{n}^{i}-x_{n-q_{i}}\right\|+\left\|x_{n-q_{i}}-x_{n}\right\| \\
& \rightarrow 0(\text { as } n \rightarrow \infty)
\end{aligned}
$$

and it follows from (19), (21) and (23)

(25) $\quad\left\|T_{m}^{n} x_{n}-x_{n}\right\| \leq\left\|T_{m}^{n} x_{n}-x_{n-q_{m}}\right\|+\left\|x_{n-q_{m}}-x_{n}\right\| \rightarrow 0 \quad($ as $n \rightarrow \infty)$.

From (21), (24) and (25), for any $i=1,2, \ldots, m$, we have

$$
\begin{aligned}
\left\|x_{n}-T_{i} x_{n}\right\| \leq & \left\|x_{n}-x_{n+1}\right\|+\left\|x_{n+1}-T_{i}^{n+1} x_{n+1}\right\|+\left\|T_{i}^{n+1} x_{n+1}-T_{i}^{n+1} x_{n}\right\| \\
& +\left\|T_{i}^{n+1} x_{n}-T_{i} x_{n}\right\| \\
\leq & \left\|x_{n}-x_{n+1}\right\|+\left\|x_{n+1}-T_{i}^{n+1} x_{n+1}\right\|+\left(1+v_{n}\right)\left\|x_{n+1}-x_{n}\right\| \\
& +\left(1+v_{1}\right)\left\|T_{i}^{n} x_{n}-x_{n}\right\| \\
= & \left(2+v_{n}\right)\left\|x_{n}-x_{n+1}\right\|+\left\|x_{n+1}-T_{i}^{n+1} x_{n+1}\right\|
\end{aligned}
$$




$$
+\left(1+v_{1}\right)\left\|T_{i}^{n} x_{n}-x_{n}\right\| \rightarrow 0 \quad(\text { as } n \rightarrow \infty) .
$$

Theorem 3. Let $E$ be a uniformly convex Banach space satisfying Opial's condition, $K$ be a nonempty closed convex subset of $E,\left\{T_{i}\right\}_{i=1}^{m}$ be a family of asymptotically quasi-nonexpansive self-mappings of $K$ with sequences (respectively) $\left\{u_{n}^{i}\right\}_{i=1}^{m} \subset[0, \infty)$ such that $\sum_{n=1}^{\infty} u_{n}^{i}<+\infty$. If the iteration sequence $\left\{x_{n}\right\}$ is defined by (2) satisfying $\left\{\beta_{n}^{i}\right\}_{i=1}^{m} \subset[\delta, 1-\delta]$ with $\delta \in(0,1)$, then $\left\{x_{n}\right\}$ converges weakly to a common fixed point of $\left\{T_{i}\right\}_{i=1}^{m}$ in $K$.

Proof. By using the same proof as in Theorem 2, it can be shown that for any $i=1,2, \ldots, m$

$$
\lim _{n \rightarrow \infty}\left\|x_{n}-T_{i} x_{n}\right\|=0 .
$$

So $I-T_{i}$ is semi-closed at 0 .

Since $E$ is uniformly convex and $\left\{x_{n}\right\}$ is bounded, we may assume that $x_{n} \rightarrow u$ as $n \rightarrow \infty$, without loss of generality. By Lemma 5 , we have $u \in F$. Suppose that subsequences $\left\{x_{n_{k}}\right\}$ and $\left\{x_{m_{k}}\right\}$ of $\left\{x_{n}\right\}$ converge weakly to $u$ and $v$, respectively. From Lemma $5, u, v \in F$. By Lemma $3, \lim _{n \rightarrow \infty}\left\|x_{n}-u\right\|$ and $\lim _{n \rightarrow \infty}\left\|x_{n}-v\right\|$ exist. It follows from Lemma 6 that $u=v$. Therefore $\left\{x_{n}\right\}$ converges weakly to a common fixed point of $\left\{T_{i}\right\}_{i=1}^{m}$.

Theorem 4. Let $E$ be a uniformly convex Banach space, $K$ be a nonempty closed convex subset of $E,\left\{T_{i}\right\}_{i=1}^{m}$ be a family of asymptotically quasi-nonexpansive self-mappings of $K$ with sequences $\left\{u_{n}^{i}\right\}_{i=1}^{m} \subset[0, \infty)$ such that $\sum_{n=1}^{\infty} u_{n}^{i}<$ $+\infty$. If the iteration sequence $\left\{x_{n}\right\}$ is defined by (2) satisfying $\left\{\beta_{n}^{i}\right\}_{i=1}^{m} \subset$ $[\delta, 1-\delta]$ with $\delta \in(0,1)$, and $\left\{T_{i}\right\}_{i=1}^{m}$ satisfies condition $(B)$ with respect to the sequence $\left\{x_{n}\right\}$, then $\left\{x_{n}\right\}$ converges strongly to a common fixed point of $\left\{T_{i}\right\}_{i=1}^{m}$ in $K$.

Proof. It follows from Theorem 2 that for any $i=1,2, \ldots, m$

$$
\lim _{n \rightarrow \infty}\left\|x_{n}-T_{i} x_{n}\right\|=0 .
$$

Since $\left\{T_{i}\right\}_{i=1}^{m}$ satisfies condition $(B)$ with respect to the sequence $\left\{x_{n}\right\}$, then there exists a nondecreasing function $f:[0, \infty) \rightarrow[0, \infty)$ with $f(0)=0, f(r)>$ 0 for all $r \in(0, \infty)$, such that for all $x_{n} \in K$

$$
f\left(d\left(x_{n}, F\right)\right) \leq \max _{0 \leq i \leq m}\left\|x_{n}-T_{i} x_{n}\right\| .
$$

So $\lim _{n \rightarrow \infty} f\left(d\left(x_{n}, F\right)\right)=0$, that is $\lim _{n \rightarrow \infty} d\left(x_{n}, F\right)=0$. By Theorem 1 , we obtain $\left\{x_{n}\right\}$ converges strongly to some $p \in F$.

Corollary 5. Let $E$ be a uniformly convex Banach space, $K$ is a nonempty closed convex subset of $E,\left\{T_{i}\right\}_{i=1}^{m}$ be a family of asymptotically quasi-nonexpansive self-mappings of $K$ with sequences $\left\{u_{n}^{i}\right\}_{i=1}^{m} \subset[0, \infty)$ such that $\sum_{n=1}^{\infty} u_{n}^{i}<$ 
$+\infty$. If the iteration sequence $\left\{x_{n}\right\}$ is defined as follows:

$$
\left\{\begin{aligned}
x_{j} & \in E(j=1,2, \ldots, r), \\
x_{n+1} & =\left(1-\beta_{n}^{1}\right) x_{n-1}+\beta_{n}^{1} T_{1}^{n} y_{n}^{1}, \\
y_{n}^{i} & =\left(1-\beta_{n}^{i+1}\right) x_{n-(i+1)}+\beta_{n}^{i+1} T_{i+1}^{n} y_{n}^{i+1}, i=1,2, \ldots, m-2, \\
y_{n}^{m-1} & =\left(1-\beta_{n}^{m}\right) x_{n-m}+\beta_{n}^{m} T_{m}^{n} x_{n}, 2 \leq m \leq r \leq n,
\end{aligned}\right.
$$

where $\left\{\beta_{n}^{i}\right\}_{i=1}^{m} \subset[\delta, 1-\delta]$ with $\delta \in(0,1)$.

(1) If E satisfying Opial's condition, then $\left\{x_{n}\right\}$ converges weakly to a common fixed point of $\left\{T_{i}\right\}_{i=1}^{m}$ in $K$.

(2) If $T$ satisfies condition $(B)$ with respect to the sequence $\left\{x_{n}\right\}$, then $\left\{x_{n}\right\}$ converges strongly to a common fixed point of $T$.

Proof. By taking $\left\{q_{i}\right\}_{i=1}^{m}=i$ for all $n \geq 1$ in (2), from Theorem 3 and Theorem 4 , the conclusion of the corollary follows.

Corollary 6. Let $E$ be a uniformly convex Banach space, $K$ is a nonempty closed convex subset of $E,\left\{T_{i}\right\}_{i=1}^{m}$ be a family of asymptotically quasi-nonexpansive self-mappings of $K$ with sequences $\left\{u_{n}^{i}\right\}_{i=1}^{m} \subset[0, \infty)$ such that $\sum_{n=1}^{\infty} u_{n}^{i}<$ $+\infty$. If the iteration sequence $\left\{x_{n}\right\}$ is defined as follows:

$$
\left\{\begin{aligned}
x_{1} & \in E, \\
x_{n+1} & =\left(1-\beta_{n}^{1}\right) x_{n}+\beta_{n}^{1} T_{1}^{n} y_{n}^{1}, \\
y_{n}^{1} & =\left(1-\beta_{n}^{2}\right) x_{n-1}+\beta_{n}^{2} T_{2}^{n} y_{n}^{2}, \\
\vdots & \\
y_{n}^{m-2} & =\left(1-\beta_{n}^{m-1}\right) x_{n-(m-2)}+\beta_{n}^{m-1} T_{m-1}^{n} y_{n}^{m-1}, \\
y_{n}^{m-1} & =\left(1-\beta_{n}^{m}\right) x_{n-(m-1)}+\beta_{n}^{m} T_{m}^{n} x_{n}, m \geq 1,
\end{aligned}\right.
$$

where $\left\{\beta_{n}^{i}\right\}_{i=1}^{m} \subset[\delta, 1-\delta]$ with $\delta \in(0,1)$.

(1) If E satisfying Opial's condition, then $\left\{x_{n}\right\}$ converges weakly to a common fixed point of $\left\{T_{i}\right\}_{i=1}^{m}$ in $K$.

(2) If $T$ satisfies condition $(B)$ with respect to the sequence $\left\{x_{n}\right\}$, then $\left\{x_{n}\right\}$ converges strongly to a common fixed point of $T$.

Proof. By taking $r=1, q_{1}=0$ and $\left\{q_{i}\right\}_{i=2}^{m}=i-1$ for all $n \geq 1$ in (2), from Theorem 3 and Theorem 4, the conclusion of the corollary follows. This completes the proof.

Corollary 7. Let $E$ be a uniformly convex Banach space, $K$ is a nonempty closed convex subset of $E,\left\{T_{i}\right\}_{i=1}^{m}$ be a family of nonexpansive self-mappings of $K$ with sequences $\left\{u_{n}^{i}\right\}_{i=1}^{m} \subset[0, \infty)$ such that $\sum_{n=1}^{\infty} u_{n}^{i}<+\infty$ and $F=$ $\bigcap_{i=1}^{m} F\left(T_{i}\right) \neq \emptyset$. If the iteration sequence $\left\{x_{n}\right\}$ is defined by (1) satisfying $\left\{\beta_{n}^{i}\right\}_{i=1}^{m} \subset[\delta, 1-\delta]$ with $\delta \in(0,1)$.

(1) If E satisfying Opial's condition, then $\left\{x_{n}\right\}$ converges weakly to a common fixed point of $\left\{T_{i}\right\}_{i=1}^{m}$ in $K$. 
(2) If $T$ satisfies condition $(B)$ with respect to the sequence $\left\{x_{n}\right\}$, then $\left\{x_{n}\right\}$ converges strongly to a common fixed point of $T$.

Proof. By taking $r=1,\left\{T_{i}^{n}\right\}_{i=1}^{m}=T$ and $\left\{q_{i}\right\}_{i=1}^{m}=0$ for all $n \geq 1$ in (2), we get (1), which is Rhoades and Soltuz introduced in [13]. From Theorem 3 and Theorem 4, the conclusion of the corollary follows.

\section{References}

[1] S. S. Chang, H. W. Joseph Lee, and C. K. Chan, On Reich's strong convergence theorem for asymptotically nonexpansive mappings in Banach spaces, Nonlinear Anal. 66 (2007), no. $11,2364-2374$.

[2] C. E. Chidume, Iterative algorithms for nonexpansive mappings and some of their generalizations, Nonlinear analysis and applications: to V. Lakshmikantham on his 80th birthday. Vol. 1, 2, 383-429, Kluwer Acad. Publ., Dordrecht, 2003.

[3] C. E. Chidume and B. Ali, Weak and strong convergence theorems for finite families of asymptotically nonexpansive mappings in Banach spaces, J. Math. Anal. Appl. 330 (2007), no. 1, 377-387.

[4] C. E. Chidume, J. L. Li, and A. Udomene, Convergence of paths and approximation of fixed points of asymptotically nonexpansive mappings, Proc. Amer. Math. Soc. 133 (2005), no. 2, 473-480.

[5] C. E. Chidume, E. U. Ofoedu, and H. Zegeye, Strong and weak convergence theorem for asymptotically nonexpansive mappings, J. Math. Anal. Appl. 280 (2003), no. 2, 364-374.

[6] C. E. Chidume and N. Shahzad, Strong convergence of an implicit iteration process for a finite family of nonexpansive mappings, Nonlinear Anal. 62 (2005), no. 6, 1149-1156.

[7] Y. J. Cho, H. Y. Zhou, and G. Guo, Weak and strong convergence theorems for threestep iterations with errors for asymptotically nonexpansive mappings, Comput. Math. Appl. 47 (2004), no. 4-5, 707-717.

[8] K. Goebel and W. A. Kirk, A fixed point theorem for asymptotically nonexpansive mappings, Proc. Amer. Math. Soc. 35 (1972), 171-174.

[9] A. R. Khan and M. A. Ahmed, Convergence of a general iterative scheme for a finite family of asymptotically quasi-nonexpansive mappings in convex metric spaces and applications, J. Appl. Math. Comput. 59 (2010), no. 8, 2990-2995.

[10] A. R. Khan, A. A. Domlo, and H. Fukhar-ud-din, Common fixed points Noor iteration for a finite family of asymptotically quasi-nonexpansive mappings in Banach spaces, J. Math. Anal. Appl. 341 (2008), no. 1, 1-11.

[11] H. Kiziltunc and S. Temir, Convergence theorems by a new iteration process for a finite family of nonself asymptotically nonexpansive mappings with errors in Banach spaces, J. Appl. Math. Comput. 61 (2011), no. 9, 2480-2489.

[12] M. O. Osilike and S. C. Aniagbosor, Weak and strong convergence theorems for fixed points of asymptotically nonexpansive mappings, Math. Comput. Modelling 32 (2000), no. 10, 1181-1191.

[13] B. E. Rhoades and S. M. Soltuz, The equivalence between Mann-Ishikawa iterations and multistep iteration, Nonlinear Anal. 58 (2004), no. 1-2, 219-228.

[14] J. Schu, Weak and strong convergence to fixed points of asymptotically nonexpansive mappings, Bull. Austral. Math. Soc. 43 (1991), no. 1, 153-159.

[15] S. Suantai, Weak and strong convergence criteria of Noor iterations for asymptotically nonexpansive mappings, J. Math. Anal. Appl. 311 (2005), no. 2, 506-517.

[16] Z. H. Sun, Strong convergence of an implicit iteration process for a finite family of asymptotically quasi-nonexpansive mappings, J. Math. Anal. Appl. 286 (2003), no. 1, 351-358. 
[17] H. K. Xu, Inequalities in Banach spaces with applications, Nonlinear Anal. 16 (1991), no. $12,1127-1138$.

[18] H. K. Xu and R. Ori, An implicit iterative process for nonexpansive mappings, Numer. Funct. Anal. Optim. 22 (2001), 767-773.

[19] İ. Yildlrlm and M. Özdemir, A new iterative process for common fixed points of finite families of non-self-asymptotically non-expansive mappings, Nonlinear Analy. 71 (2009), no. 3-4, 991-999.

[20] Y. Zhou and S. S. Chang, Convergence of implicit iteration process for a finite family of asymptotically nonexpansive mappings in Banach spaces, Numer. Funct. Anal. Appl. Optim. 23 (2002), no. 7-8, 911-921.

JUAN XIAO

School of Mathematics AND Statistics

SOUTHWEST UNIVERSITY

Chongqing 400715, P. R. China

E-mail address: juanxiao@swu.edu.cn

LEI DENG

School of Mathematics and Statistics

SOUTHWEST UNIVERSITY

Chongqing 400715, P. R. China

E-mail address: denglei@swu.edu.cn

Ming-GE YANG

College of Mathematics Science

LuOyang Normal University

Luoyang, Henan 471022, P. R. China

E-mail address: yang_mingge@sina.com 